\title{
Analytical Least-Squares Solution for 3D Lidar-Camera Calibration
}

\author{
Faraz M. Mirzaei, Dimitrios G. Kottas, and Stergios I. Roumeliotis
}

\begin{abstract}
This paper addresses the problem of estimating the intrinsic parameters of the 3D Velodyne lidar while at the same time computing its extrinsic calibration with respect to a rigidly connected camera. Existing approaches to solve this nonlinear estimation problem are based on iterative minimization of nonlinear cost functions. In such cases, the accuracy of the resulting solution hinges on the availability of a precise initial estimate, which is often not available. In order to address this issue, we divide the problem into two least-squares sub-problems, and analytically solve each one to determine a precise initial estimate for the unknown parameters. We further increase the accuracy of these initial estimates by iteratively minimizing a batch nonlinear least-squares cost function. In addition, we provide the minimal observability conditions, under which, it is possible to accurately estimate the unknown parameters. Experimental results consisting of photorealistic 3D reconstruction of indoor and outdoor scenes are used to assess the validity of our approach.
\end{abstract}

\section{Introduction and Related Work}

As demonstrated in the DARPA Urban Challenge, commercially available highspeed 3D lidars, such as the Velodyne, have made autonomous navigation and mapping within dynamic environment possible. In most applications, however, another sensor is employed in conjunction with the 3D lidar to assist in localization and place recognition. In particular, spherical cameras are often used to provide visual cues and to construct photorealistic maps of the environment. In these scenarios, accurate extrinsic calibration of the six degrees of freedom (d.o.f.) transformation

Authors are with the University of Minnesota, Minneapolis, MN 55455

Emails:\{faraz|dkottas|stergios\}@cs.umn. edu.

This work was supported by the University of Minnesota (DTC), and the National Science Foundation (IIS-0643680, IIS-0811946, IIS-0835637) 
between the two sensors is a prerequisite for optimally combining their measurements.

Several methods exist for calibrating a 2D laser scanner with respect to a camera. The work of Zhang and Pless [17] relies on the observation of a planar checkerboard by both sensors. In particular, corners are detected in images and planar surfaces are extracted from the laser measurements. The image features are used to determine the normal vector and distance of the planes where the laser-scan endpoints lie. Using this geometric constraint, the estimation of the transformation between the two sensors is formulated as a non-linear least-squares problem and solved iteratively. A simplified linear least-squares solution is also provided to initialize the iterative nonlinear algorithm. More recently, Naroditsky et al. have presented a minimal approach for calibrating a $2 \mathrm{D}$ laser scanner with respect to a camera, using only six measurements of a planar calibration board [6]. The computed transformation is then used in conjunction with RANSAC to initialize an iterative least-squares refinement.

The existing 2D laser scanner-camera calibration methods are extended to 3D lidars in $[16,7]$. In both works, a geometric constraint similar to that of [17] is employed to form a nonlinear least-squares cost function which is iteratively minimized to estimate the lidar-camera transformation. An initial estimate for the iterative minimization is determined based on a simplified linear least-squares method [16]. Specifically, the estimation of relative rotation and translation are decoupled, and then each of them is computed from a geometric constraint between the planar segments detected in the measurements of both the 3D lidar and the camera. An alternative 3D lidar-camera calibration approach is described in [11], where several point correspondences are manually selected in images and their associated lidar scans. Then, the PnP algorithm of [9] is employed to find the transformation between the camera and the 3D lidar based on these point correspondences. In a different approach, presented in [13], the structural edges extracted from 3D lidar scans are matched with the vanishing points of the corresponding $2 \mathrm{D}$ images to compute a coarse 3D lidar-camera transformation, followed by an iterative least-squares refinement.

The main limitation of the above methods is that they assume the 3D lidar to be intrinsically calibrated. If the lidar's intrinsic calibration is not available or sufficiently accurate, then the calibration accuracy as well as the performance of subsequent lidar-camera data fusion significantly degrades. In [7], this issue is partially addressed for the Velodyne 3D lidar by first calibrating only some of its intrinsic parameters. However, this intrinsic calibration procedure is also iterative, and no method is provided for initializing it. While several of the intrinsic parameters of a lidar may be initialized using the technical drawings of the device (if available), other parameters, such as the offset in the range measurements induced by the delay in the electronic circuits, cannot be determined in this way.

To address these limitations, in this work we propose a novel algorithm for joint estimation of both the intrinsic parameters of the Velodyne 3D lidar and the lidarcamera transformation. Specifically, we use measurements of a calibration plane at various configurations to establish geometric constraints between the lidar's in- 
trinsic parameters and the lidar-camera 6 d.o.f. relative transformation. We process these measurement constraints to estimate the calibration parameters as follows: First, we analytically compute an initial estimate for the intrinsic and extrinsic calibration parameters in two steps. Subsequently, we employ a batch iterative (nonlinear) least-squares method to refine the accuracy of the estimated parameters.

In particular, to analytically compute an initial estimate, we relax the estimation problem by seeking to determine the transformation between the camera and each one of the conic laser scanners within the Velodyne, along with its intrinsic parameters. As a first step, we formulate a nonlinear least-squares problem to estimate the 3 d.o.f. rotation between each conic laser scanner and the camera, as well as a subset of the laser scanner's intrinsic parameters. The optimality conditions of this nonlinear least-squares form a system of polynomial equations, which we solve analytically using an algebraic-geometry approach to find all its critical points. Amongst these, the one that minimizes the least-squares cost function corresponds to the global minimum and provides us with the initial estimates for the relative rotation and the first set of intrinsic lidar parameters. In the next step, we use a linear least-squares algorithm to compute the initial estimate for the relative translation between the camera and the conic laser scanners, and the remaining intrinsic parameters.

Once all initial estimates are available, we finally perform a batch iterative jointoptimization of the lidar-camera transformation and the lidar's intrinsic parameters. As part of our contributions, we also study the observability properties of the problem and present the minimal necessary conditions for concurrently estimating the lidar's intrinsic parameters and the lidar-camera transformation. These observability conditions provide a guideline for designing high-accuracy calibration procedures. Our experimental results demonstrate that our proposed method significantly improves the accuracy of the intrinsic calibration parameters of the Velodyne lidar, as well as, the lidar-camera transformation.

\section{Problem Formulation}

The Velodyne HDL-64E lidar consists of 64 conic laser scanners mounted on a rotating head so that they span a $360^{\circ}$ panoramic (azimuth) view (see Fig. 1). Each laser scanner has a horizontal offset from the axis of rotation, and a vertical offset from adjacent laser scanners. Additionally, each laser scanner points to a different elevation angle, such that, collectively, all the laser scanners cover a $27^{\circ}$ vertical field of view. Therefore, once the lidar's head completes a full rotation, each laser scanner has swept a cone in space specified by its elevation angle. Let $\{L\}$ be the lidar's fixed frame of reference whose $z$-axis is the axis of rotation of the sensor's head (see Fig. 1). Also, let $\left\{L_{i}\right\}, i=1, \ldots, 64$, be the coordinate frame corresponding to the $i$-th laser scanner, such that its origin is at the center of the associated cone on the $z$-axis of $\{L\}$ with vertical offset $h_{i}$ from the origin of $\{L\}$, its $z$-axis aligned with that of $\{L\}$, and its $x$-axis defining an angle $\theta_{o i}$ with the $x$-axis of $\{L\}$. We determine the direction of the $k$-th shot of the $i$-th laser beam from its corresponding elevation 


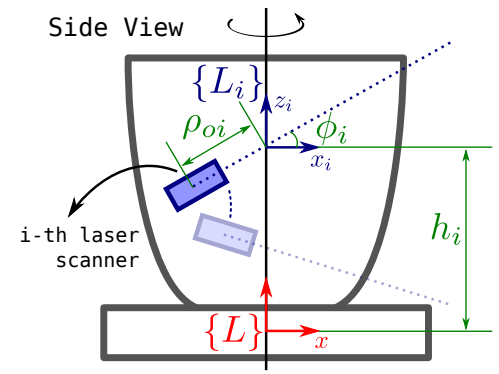

Top View

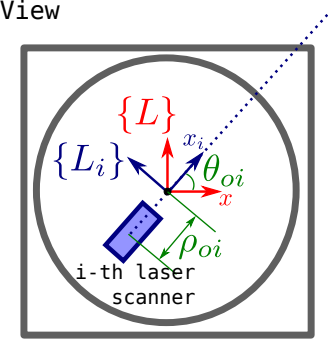

Fig. 1 Internal structure of the Velodyne 3D lidar. The total number of laser beams is 64 and they span a vertical field of view of $27^{\circ}$. The intrinsic parameters of the Velodyne describe the measurements of each laser scanner in its coordinate frame, $\left\{L_{i}\right\}$, and the transformation between the Velodyne's fixed coordinate frame, $\{L\}$, and $\left\{L_{i}\right\}$. Note that besides the physical offset of the laser scanners from the axis of rotation, the value of $\rho_{o i}$ depends on the delay in the electronic circuits of the lidar.

angle, $\phi_{i}$, and azimuth measurement, $\theta_{i k}$, and denote it with: ${ }^{1}$

$$
{ }^{L_{i}} \overline{\mathbf{p}}_{k} \triangleq\left[\begin{array}{c}
\cos \phi_{i} \cos \theta_{i k} \\
\cos \phi_{i} \sin \theta_{i k} \\
\sin \phi_{i}
\end{array}\right] .
$$

The distance measured by the $k$-th shot of the $i$-th laser scanner is represented by $\rho_{i k}$. The real distance to the object that reflects the $k$-th shot of the $i$-th laser beam is $\alpha_{i}\left(\rho_{i k}+\rho_{o i}\right)$, where $\alpha_{i}$ is the scale factor, and $\rho_{o i}$ is the range offset due to the delay in the electronic circuits of the lidar and the horizontal offset of each laser scanner from its cone's center. In this way, the position of the $k$-th point measured by the $i$-th laser scanner is described by

$$
{ }^{L_{i}} \mathbf{p}_{i k}=\alpha_{i}\left(\rho_{i k}+\rho_{o i}\right)^{L_{i}} \overline{\mathbf{p}}_{k} .
$$

The transformation between $\left\{L_{i}\right\}$ and $\{L\}$ (i.e., $h_{i}$ and $\theta_{o i}$ ), the scale $\alpha_{i}$, offset $\rho_{o i}$, and elevation angle $\phi_{i}$, for $i=1, \ldots, 64$, comprise the intrinsic parameters of the lidar that must be precisely known for any application, including photorealistic reconstruction of the surroundings. Since the intrinsic parameters supplied by the manufacturer may not be accurate,$^{2}$ in this work we estimate them along with the transformation with respect to a camera.

\footnotetext{
${ }^{1}$ Throughout this paper, ${ }^{A} \mathbf{p}$ denotes the expression of a vector $\mathbf{p}$ with respect to frame $\{A\}$ and $\overline{\mathbf{p}}$ is the corresponding unit vector. ${ }_{B}^{A} \mathbf{C}$ is the rotation matrix rotating vectors from frame $\{B\}$ to frame $\{A\}$, and ${ }^{A} \mathbf{t}_{B}$ is the position of the origin of $\{B\}$ in $\{A\} . \mathbf{I}_{n}$ is the $n \times n$ identity matrix, and $\mathbf{0}_{m \times n}$ is the $m \times n$ matrix of zeros.

${ }^{2}$ Note that when the technical drawings of the lidar are available, an initial estimate for $h_{i}, \theta_{o i}$, and $\phi_{i}$ can be readily obtained. Computing an initial estimate for $\rho_{o i}$ and $\alpha_{i}$, however, is significantly more challenging even for the manufacturer, since their values do not solely depend on the physical dimensions of the device.
} 


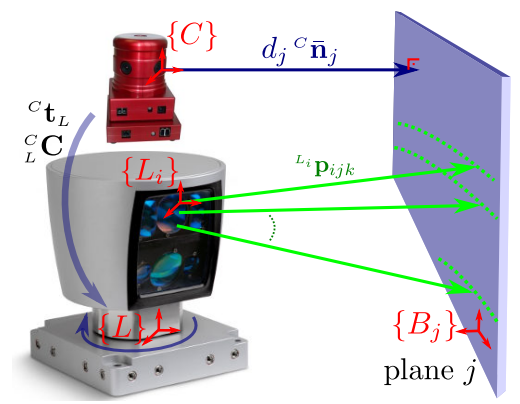

Fig. 2 Geometric constraint between the $j$-th plane, the Ladybug $\{C\}$, and the $i$-th laser scanner, $\left\{L_{i}\right\}$. Each laser beam is described by a vector ${ }^{L_{i}} \mathbf{p}_{i j k}$. The plane is described by its normal vector ${ }^{C} \overline{\mathbf{n}}_{j}$ and its distance $d_{j}$ both expressed with respect to the Ladybug.

The Ladybug2 spherical vision system consists of six rigidly-connected calibrated cameras equipped with wide-angle lenses (see Fig. 2). The extrinsic transformations between the different cameras are provided by the manufacturer with high accuracy. Therefore, the measurements from any of the cameras can be easily transformed to the Ladybug's fixed frame of reference, denoted as $\{C\}$.

The Ladybug is rigidly connected to the lidar, and our objective is to determine the 6 d.o.f. relative transformation between the two, as well as the intrinsic parameters of the lidar. For this purpose, we employ a planar calibration board with fiducial markers, at $M$ different configurations to establish geometric constraints between the measurements of the lidar and the Ladybug, their relative transformation, and the lidar's intrinsic parameters.

Specifically, at the $j$-th plane configuration, $j=1, \ldots, M$, the fiducial markers whose positions are known with respect to the calibration board's frame of reference $\left\{B_{j}\right\}$, are first detected in the Ladybug's image. The 6 d.o.f. transformation between $\{C\}$ and $\left\{B_{j}\right\}$ is then computed using a PnP algorithm [9], from which the normal vector and the distance of the target plane in the Ladybug's frame are extracted as:

$$
\begin{aligned}
& { }^{C} \overline{\mathbf{n}}_{j} \triangleq{ }_{B_{j}}^{C} \mathbf{C}\left[\begin{array}{lll}
0 & 0 & -1
\end{array}\right]^{T} \\
& d_{j} \triangleq{ }^{C} \overline{\mathbf{n}}_{j}^{T C} \mathbf{t}_{B_{j}}
\end{aligned}
$$

where ${ }_{B j}^{C} \mathbf{C}$ and ${ }^{C} t_{B_{j}}$ represent the relative rotation and translation between the Ladybug and the calibration board at the $j$-th configuration. Consequently, in the absence of noise, any point ${ }^{C} \mathbf{p}$ that lies on the $j$-th plane satisfies:

$$
{ }^{C} \overline{\mathbf{n}}_{j}^{T}{ }^{C} \mathbf{p}-d_{j}=0 .
$$

We now turn our attention to the lidar point measurements reflected from the $j$-th calibration plane and identified based on the depth discontinuity. Let us denote such points as ${ }^{L_{i}} \mathbf{p}_{i j k}, k=1 \ldots, N_{i j}$, measured by the lidar's $i$-th laser scanner [see (2)]. Transforming these points to the Ladybug's frame, and substituting them in (5) yields: 


$$
\begin{array}{r}
{ }^{C} \overline{\mathbf{n}}_{j}^{T}\left({ }_{L_{i}}^{C} \mathbf{C}^{L_{i}} \mathbf{p}_{i j k}+{ }^{C} \mathbf{t}_{L_{i}}\right)-d_{j}=0 \\
\stackrel{(2)}{\Longrightarrow} \alpha_{i}\left(\rho_{i j k}+\rho_{o i}\right)^{C} \overline{\mathbf{n}}_{j L_{i}}^{T} \mathbf{C}^{L_{i}} \overline{\mathbf{p}}_{i j k}+{ }^{C} \overline{\mathbf{n}}_{j}^{T}{ }^{C} \mathbf{t}_{L_{i}}-d_{j}=0
\end{array}
$$

where ${ }_{L_{i}}^{C} \mathbf{C}$ and ${ }^{C} \mathbf{t}_{L_{i}}$ are the relative rotation and translation between the Ladybug and the $i$-th laser scanner.

In addition to the camera and laser scanner measurements, the following constraints can also be used to increase the accuracy of the calibration process. Specifically, since the $z$-axis of $\left\{L_{i}\right\}$ is aligned with the $z$-axis of $\{L\}$, while their $x$-axes form an angle $\theta_{o i}$, the following constraint holds for all ${ }_{L_{i}}^{C} \mathbf{C}$ :

$$
{ }_{L_{i}}^{C} \mathbf{C}={ }_{L}^{C} \mathbf{C C}_{z}\left(\theta_{o i}\right)
$$

where $\mathbf{C}_{z}\left(\theta_{o i}\right)$ represents a rotation around the $z$-axis by an angle $\theta_{o i}$. Additionally, the origin of each laser-scanner frame lies on the $z$-axis of $\{L\}$ with vertical offset of $h_{i}$ from the origin of $\{L\}$, resulting in the following constraint:

$$
{ }_{L}^{C} \mathbf{C}^{T}\left({ }^{C} t_{L_{i}}-{ }^{C} t_{L}\right)=\left[\begin{array}{lll}
0 & 0 & h_{i}
\end{array}\right]^{T}
$$

In the presence of noise, the geometric constraint in (7) is not exactly satisfied. Therefore, to estimate the unknown parameters, we form a constrained nonlinear least-squares cost function from the residuals of this geometric constraint over all point and plane observations [see (22)]. In order to minimize this least-squares cost, one has to employ iterative minimizers such as the Levenberg-Marquardt [8], that require a precise initial estimate to ensure convergence. To provide accurate initialization, in the next three sections we present a novel analytical method to estimate the lidar-Ladybug transformation and all intrinsic parameters of the lidar (except the elevation angle $\phi_{i}$ which is precisely known from the manufacturer). In order to reduce the complexity of the initialization process, we temporarily drop the constraints in (8) and (9) and seek to determine the transformation between the camera and each of the laser scanners (along with each scanner's intrinsic parameters) independently. Once an accurate initial estimate is computed, we lastly perform an iterative non-linear least-squares refinement that explicitly considers (8) and (9), and increases the calibration accuracy (see Section 2.4).

\subsection{Analytical Estimation of Offset and Relative Rotations}

Note that the term ${ }^{C} \overline{\mathbf{n}}_{j}^{T} C_{t_{L_{i}}}-d_{j}$ in (7) is constant for all points $k$ of the $i$-th laser scanner that hit the calibration plane at its $j$-th configuration. Therefore, subtracting two constraints of the form (7) for the points ${ }^{L_{i}} \mathbf{p}_{i j k}$ and ${ }^{L_{i}} \mathbf{p}_{i j l}$, and dividing the result by the nonzero scale, $\alpha_{i}$, yields:

$$
{ }^{C} \overline{\mathbf{n}}_{j L_{i}}^{T C} \mathbf{C}\left(\mathbf{u}_{j k l}^{i}+\rho_{o i} \mathbf{v}_{j k l}^{i}\right)=0
$$


where $\mathbf{u}_{j k l}^{i} \triangleq \rho_{i j k}{ }^{L_{i}} \overline{\mathbf{p}}_{i j k}-\rho_{i j l}{ }^{L_{i}} \overline{\mathbf{p}}_{i j l}$ and $\mathbf{v}_{j k l}^{i} \triangleq{ }^{L_{i}} \overline{\mathbf{p}}_{i j k}-{ }^{L_{i}} \overline{\mathbf{p}}_{i j l}$. Note that the only unknowns in this constraint are the relative rotation of the $i$-th laser scanner with respect to the Ladybug, ${ }_{L_{i}}^{C} \mathbf{C}$, and its offset, $\rho_{o i}$. Let us express the former, ${ }_{L_{i}}^{C} \mathbf{C}$, using the Cayley-Gibbs-Rodriguez (CGR) parameterization [12], i.e.,

$$
{ }_{L_{i}}^{C} \mathbf{C}(\mathbf{s})=\frac{\overline{\mathbf{C}}\left(\mathbf{s}_{i}\right)}{1+\mathbf{s}_{i}^{T} \mathbf{s}_{i}}, \quad \overline{\mathbf{C}}\left(\mathbf{s}_{i}\right) \triangleq\left(\left(1-\mathbf{s}_{i}^{T} \mathbf{s}_{i}\right) \mathbf{I}_{3}+2\left\lfloor\mathbf{s}_{i} \times\right\rfloor+2 \mathbf{s}_{i} \mathbf{s}_{i}^{T}\right)
$$

where $\mathbf{s}_{i}^{T}=\left[\begin{array}{lll}s_{i 1} & s_{i 2} & s_{i 3}\end{array}\right]$ is the vector of CGR parameters that represent the relative orientation of the $i$-th laser scanner with respect to the Ladybug, and $\lfloor\mathbf{s} \times\rfloor$ is the corresponding skew-symmetric matrix [12]. Substituting (11) in (10), and multiplying both sides with the nonzero term $1+\mathbf{s}_{i}^{T} \mathbf{s}_{i}$ yields:

$$
{ }^{C} \overline{\mathbf{n}}_{j}^{T} \overline{\mathbf{C}}\left(\mathbf{s}_{i}\right)\left(\mathbf{u}_{j k l}^{i}+\rho_{o i} \mathbf{v}_{j k l}^{i}\right)=0
$$

This algebraic constraint holds exactly in the absence of noise. In that case, the method presented in Section 2.5 can be employed to recover the unknowns given the minimum number of measurements. In the presence of noise, however, (12) becomes:

$$
{ }^{C} \overline{\mathbf{n}}_{j}^{T} \overline{\mathbf{C}}\left(\mathbf{s}_{i}\right)\left(\mathbf{u}_{j k l}^{i}+\rho_{o i} \mathbf{v}_{j k l}^{i}\right)=\eta_{j k l}^{i}
$$

where $\eta_{j k l}^{i}$ is a nonzero residual. In this case, we estimate $\mathbf{s}_{i}$ and $\rho_{o i}$ by solving the following nonlinear least-squares problem:

$$
\hat{\mathbf{s}}_{i}, \hat{\rho}_{o i}=\min _{\mathbf{s}_{i}, \rho_{o i}} J_{i}, \quad J_{i} \triangleq \frac{1}{2} \sum_{j=1}^{M} \sum_{k=1}^{\frac{N_{i j}}{2}} \sum_{l=\frac{N_{i j}}{2}+1}^{N_{i j}}\left({ }^{C} \overline{\mathbf{n}}_{j}^{T} \overline{\mathbf{C}}\left(\mathbf{s}_{i}\right)\left(\mathbf{u}_{j k l}^{i}+\rho_{o i} \mathbf{v}_{j k l}^{i}\right)\right)^{2}
$$

where, without loss of generality, we have assumed $N_{i j}$ is even. Note that the $N_{i j}$ points from the $i$-th laser scanner, and the $j$-th configuration of the calibration plane are divided into two mutually exclusive groups so as to ensure that each point appears in the least-squares cost only once and hence avoid noise correlations.

When a sufficient number of plane configurations are observed, we employ a recently proposed algebraic method to directly solve this nonlinear least-squares problem without requiring initialization [14]. Specifically, we first form the following polynomial system describing the optimality conditions of (14):

$$
f_{i \ell}=\frac{\partial J_{i}}{\partial s_{i \ell}}=0, \ell=0, \ldots, 3, \text { and } s_{i 0} \triangleq \rho_{o i} .
$$

Note that the cost function in (14) is a polynomial of degree six in the elements of $\mathbf{s}_{i}$ and $\rho_{o i}$. Therefore, (15) consists of four polynomials of degree five in four variables. This polynomial system has 243 solutions that comprise the critical points of the least-squares cost function $J_{i}$, and can be computed using the eigenvalue decomposition of the so-called multiplication matrix (see Section 2.2). The globally opti- 
mal solution of the least-squares problem is the critical point that minimizes (14), and it is selected through direct evaluation of the cost function $J_{i}$. We point out that the computational complexity of solving (15) and finding the global minimum does not increase with the addition of measurements, since the degree and number of polynomials expressing the optimality conditions are fixed regardless of the number of calibration-plane configurations and laser-scanner points reflected from them. Moreover, computing the contribution of all points to the coefficients of the polynomials $f_{i \ell}, \ell=0, \ldots, 3$, increases only linearly with the number of measurements.

\subsection{Polynomial System Solver}

In order to solve the polynomials describing the optimality conditions of (15), we compute the multiplication matrix, a generalization of the companion matrix to systems of multivariate polynomial equations, whose eigenvalues are the roots of the associated polynomial system [1]. In the following, we briefly describe an efficient method for computing the multiplication matrix. For a detailed discussion on solving systems of polynomial equations, we refer the interested reader to [5].

Let us denote a monomial in $\mathbf{x}=\left[x_{1} \cdots x_{n}\right]^{T}$ by $x^{\gamma} \triangleq x_{1}^{\gamma_{1}} x_{2}^{\gamma_{2}} \cdots x_{n}^{\gamma_{n}}, \gamma_{i} \in \mathbb{Z}_{\geq 0}$, with degree $\sum_{i=1}^{n} \gamma_{i}$. A polynomial of degree $d$ in $\mathbf{x}$ is denoted by $f=\mathbf{c}^{T} \mathbf{x}_{d}$ where $\mathbf{x}_{d}$ is the $\left(\begin{array}{c}n+d \\ n\end{array}\right)$-dimensional vector of monomials of degree up to and including $d$, and $\mathbf{c}$ is the vector of coefficients of equal size. We assume that the given system of equations has $n$ polynomials, denoted by $f_{i}=\mathbf{c}_{i}^{T} \mathbf{x}_{d_{i}}=0, i=1, \ldots, n$, each of them with degree $d_{i}$. The total degree of the polynomial system is $d \triangleq \max _{i} d_{i}$. By padding the coefficient vectors of $f_{i}$ 's with zeros, and stacking them together in $\mathbf{C}$, we can present the polynomial system in the matrix form of $\mathbf{C} \mathbf{x}_{d}=\mathbf{0}$.

A system of polynomial equations defines an ideal $I$ as the set of all the polynomials that can be generated as $\sum_{i} f_{i} h_{i}$ where $h_{i}$ is any polynomial in $\mathbf{x}$. Clearly the elements of the ideal become zero at the solutions of the original (generator) polynomial system. The Gröbner basis $G \triangleq\left\langle g_{1}, \ldots g_{t}\right\rangle$ of an ideal is a finite subset of the ideal such that (i) the remainder of the division of any polynomial to it is unique, (ii) any polynomial whose division by the Gröbner basis results in zero remainder, is a member of the associated ideal. The first property can be expressed as: $\varphi(\mathbf{x})=r(\mathbf{x})+\sum_{i=1}^{t} g_{i}(\mathbf{x}) h_{i}(\mathbf{x})$ where $\varphi$ is any polynomial in $\mathbf{x}, h_{i}$ 's are the quotient polynomials, and $r$ is the unique remainder. We hereafter use the name "remainder" as the remainder of the division of a polynomial by the Gröbner basis. The Gröbner basis for an ideal generated from polynomials with integer or rational numbers can be computed using implementations of the so-called Buchberger's algorithm [5] on symbolic software packages such as Macaulay2 or Maple. Computation of the Gröbner basis for polynomials with floating-point coefficients is much more difficult due to quick accumulation of round-off errors in the Buchberger's algorithm.

The remainders of the polynomials that are not in an ideal are instrumental in finding the solutions (i.e., variety) of that ideal. It can be shown that all such remainders can be expressed as a linear combination of a specific (unique) group of 
monomials that comprise the so-called normal set [5]. The normal set can be easily obtained from the Gröbner basis of an ideal, and under mild conditions, ${ }^{3}$ its cardinality equals the number of solutions (real and complex) of the ideal, and it will contain the monomial $1[5, \mathrm{p} .43]$. The important point here is that the normal set is generically fixed across different instantiations of the polynomials. Therefore, we can compute the normal set of an instance of the problem (e.g., integer or rational coefficients) and use it when the coefficients are floating point.

Let us assume that the cardinality of the normal set is $s$, and represent its monomials in a vector form $\mathbf{x}_{B}$. Then multiplication of $\mathbf{x}_{B}$ with a generic polynomial $\varphi(\mathbf{x})$ yields:

$$
\boldsymbol{\varphi}(\mathbf{x}) \cdot \mathbf{x}_{B}=\mathbf{M}_{\varphi} \mathbf{x}_{B}+\left[\begin{array}{ccc}
h_{11} & \cdots & h_{1 t} \\
\vdots & & \vdots \\
h_{s 1} & \cdots & h_{s t}
\end{array}\right]\left[\begin{array}{c}
g_{1} \\
\vdots \\
g_{t}
\end{array}\right]
$$

where $h_{i j}$ 's are polynomials in $\mathbf{x}$, and $g_{i}$ 's are the elements of the Gröbner basis. In this expression, $\mathbf{M}_{\varphi}$ is called the multiplication matrix associated with $\varphi$. This relationship holds since the remainder of any polynomial (including $x^{\gamma} \varphi(\mathbf{x}), x^{\gamma} \in \mathbf{x}_{B}$ ) can be written as a linear combination of $\mathbf{x}_{B}$. Now, if we evaluate (16) at $\mathbf{x}=\mathbf{p}$, a solution of the ideal, all $g_{i}$ 's become zero, and we get $\varphi(\mathbf{p}) \cdot \mathbf{p}_{B}=\mathbf{M}_{\varphi} \mathbf{p}_{B}$, where $\mathbf{p}_{B}$ is $\mathbf{x}_{B}$ evaluated at $\mathbf{p}$. Clearly, $\mathbf{p}_{B}$ is an eigenvector of $\mathbf{M}_{\varphi}$, and $\varphi(\mathbf{p})$ is the associated eigenvalue. Therefore, if we select $\varphi(\mathbf{x})$ equal to one of the variables (e.g., $\left.x_{i}\right)$, we can read off the $x_{i}$-coordinate of the solutions as the eigenvalues of $\mathbf{M}_{\varphi}$. Furthermore, depending on the ordering of the monomials when computing the Gröbner basis, $\mathbf{x}_{B}$ may include all first-order monomials $x_{1}, \ldots, x_{n}$. In that case, one can simultaneously read off all the coordinates of the solutions, for an arbitrary choice of $\varphi$, as long as it is nonzero and distinct at each solution of the ideal.

When the Gröbner basis is available (such as in polynomial systems with integer coefficients), one can use it directly to compute remainders of $\varphi(\mathbf{x}) \cdot \mathbf{x}_{B}$, and construct $\mathbf{M}_{\varphi}$. This is not possible, however, when working with polynomials with floating-point coefficients. Therefore we employ the method proposed in [3] to compute $\mathbf{M}_{\varphi}$. We first note that some of the monomials of $\varphi(\mathbf{x}) \cdot \mathbf{x}_{B}$ remain in $\mathbf{x}_{B}$, while some others do not. We form the vector $\mathbf{x}_{R}$ from the latter monomials, and write:

$$
\varphi(\mathbf{x}) \cdot \mathbf{x}_{B}=\mathbf{M}_{\varphi}^{\prime}\left[\begin{array}{l}
\mathbf{x}_{R} \\
\mathbf{x}_{B}
\end{array}\right]
$$

where $\mathbf{M}_{\varphi}^{\prime}$ is called the unreduced multiplication matrix. Our objective is to express the remainders of $\mathbf{x}_{R}$ as a linear combination of $\mathbf{x}_{B}$ without using the Gröbner basis. For this purpose, we expand each original polynomial $f_{i}$ by multiplying it with all the monomials up to degree $\ell-d_{i}$ ( $\ell$ to be determined later). Clearly all these new expanded polynomials belong to the ideal generated by the original polynomials, and they have monomials up to degree $\ell$. Thus, we can write them collectively in matrix form as $\mathbf{C}_{e} \mathbf{x}_{\ell}=0$. We reorder $\mathbf{x}_{\ell}$ and $\mathbf{C}_{e}$ as:

\footnotetext{
${ }^{3}$ These conditions are: (i) the ideal must be radical, (ii) its variety must be non-empty and zero dimensional [5]. These conditions are generally satisfied for the current problem.
} 


$$
\mathbf{C}_{e} \mathbf{x}_{\ell}=\left[\begin{array}{lll}
\mathbf{C}_{E} & \mathbf{C}_{R} & \mathbf{C}_{B}
\end{array}\right]\left[\begin{array}{l}
\mathbf{x}_{E} \\
\mathbf{x}_{R} \\
\mathbf{x}_{B}
\end{array}\right]=0
$$

where $\mathbf{x}_{E}$ are the monomials that belong neither to $\mathbf{x}_{R}$ nor to $\mathbf{x}_{B}$. Multiplying (18) with $\mathbf{N}^{T}$, the left null space of $\mathbf{C}_{E}$, and decomposing $\mathbf{N}^{T} \mathbf{C}_{R}=\mathbf{Q R}=\left[\begin{array}{ll}\mathbf{Q}_{1} & \left.\mathbf{Q}_{2}\right]\left[\mathbf{R}_{1}^{T} \mathbf{0}\right.\end{array}\right]^{T}$ using $\mathrm{QR}$ factorization, yields:

$$
\left[\begin{array}{ll}
\mathbf{N}^{T} \mathbf{C}_{R} \mathbf{N}^{T} \mathbf{C}_{B}
\end{array}\right]\left[\begin{array}{c}
\mathbf{x}_{R} \\
\mathbf{x}_{B}
\end{array}\right]=\mathbf{Q}\left[\begin{array}{cc}
\mathbf{R}_{1} & \mathbf{Q}_{1}^{T} \mathbf{N}^{T} \mathbf{C}_{B} \\
\mathbf{0} & \mathbf{Q}_{2}^{T} \mathbf{N}^{T} \mathbf{C}_{B}
\end{array}\right]\left[\begin{array}{l}
\mathbf{x}_{R} \\
\mathbf{x}_{B}
\end{array}\right]=\mathbf{0} .
$$

If $\ell$ is selected sufficiently large, $\mathbf{R}_{1}$ will be full rank [10], which allows us to solve (19) and find $\mathbf{x}_{R}$ as a function of $\mathbf{x}_{B}$, i.e., $\mathbf{x}_{R}=-\mathbf{R}_{1}^{-1} \mathbf{Q}_{1}^{T} \mathbf{N}^{T} \mathbf{C}_{B} \mathbf{x}_{B}$. Substituting this relationship in (17) yields the multiplication matrix:

$$
\mathbf{M}_{\varphi}=\mathbf{M}_{\varphi}^{\prime}\left[\begin{array}{c}
\mathbf{I}_{s} \\
-\mathbf{R}_{1}^{-1} \mathbf{Q}_{1}^{T} \mathbf{N}^{T} \mathbf{C}_{B}
\end{array}\right] .
$$

For solving equations (15), we had to expand the polynomials up to degree $\ell=15$ and arrived at a multiplication matrix $\mathbf{M}_{\varphi}$ of dimensions $243 \times 243$. Finally, we mention that it is possible to compute the multiplication matrix without explicit computation of the normal set. Further details on this subject and also on possible numerical instabilities and their remedies are given in $[3,10]$ and [15].

\subsection{Analytical Estimation of Scale and Relative Translation}

Once the relative rotation, ${ }_{L_{i}}^{C} \mathbf{C}$, and the offset, $\rho_{o i}$, of each laser scanner, $i=1, \ldots, 64$, are computed, we use linear least-squares to determine the relative translation and scale from (7). Specifically, we stack together all the measurement constraints on the $i$-th laser scanner's scale and relative translation (from different points and calibration-plane configurations), and write them in a matrix form as:

$$
\left[\begin{array}{cc}
{ }^{C} \overline{\mathbf{n}}_{1}^{T} & \left(\rho_{i 11}+\rho_{o i}\right)^{C} \overline{\mathbf{n}}_{1 L_{i}^{T}}^{C} \mathbf{C}^{L_{i}} \overline{\mathbf{p}}_{i 11} \\
{ }^{C} \overline{\mathbf{n}}_{1}^{T} & \left(\rho_{i 12}+\rho_{o i}\right)^{C} \overline{\mathbf{n}}_{1 L_{i}}^{T} \mathbf{C}^{L_{i}} \overline{\mathbf{p}}_{i 12} \\
\vdots & \vdots \\
{ }^{C} \overline{\mathbf{n}}_{M}^{T}\left(\rho_{i M N_{i M}}+\rho_{o i}\right)^{C} \overline{\mathbf{n}}_{M L_{i}}^{T}{ }^{C} \mathbf{C}^{L_{i}} \overline{\mathbf{p}}_{i M N_{i M}}
\end{array}\right]\left[\begin{array}{c}
d_{1} \\
{ }_{t_{L_{i}}} \\
\alpha_{i}
\end{array}\right]=\left[\begin{array}{c}
d_{1} \\
\vdots \\
d_{M}
\end{array}\right]
$$

Under the condition that the coefficient matrix on the left-hand side of this equality is full rank (see Section 2.5), we can easily obtain the $i$-th laser scanner's scale factor, $\alpha_{i}$, and relative translation, ${ }^{C} \mathbf{t}_{L_{i}}$, by solving (21). 


\subsection{Iterative Refinement}

Once the initial estimates for the transformation between the Ladybug and the laser scanners, and the intrinsic parameters of the lidar are known (Sections 2.1 to 2.3), we employ an iterative refinement method to enforce the constraints in (8) and (9). Specifically, we choose the coordinate frame of one of the laser scanners (e.g., the 1-st laser scanner) as the lidar's fixed coordinate frame, i.e., $\{L\}=\left\{L_{1}\right\}$. Then for $\left\{L_{i}\right\}, i=2, \ldots, 64$, we employ the estimated relative transformation with respect to the Ladybug (i.e., ${ }_{L_{i}}^{C} \mathbf{C}$ and ${ }^{C} \mathbf{t}_{L_{i}}$ ) to obtain the relative transformations between $\left\{L_{i}\right\}$ and $\{L\}$. From these relative transformations, we only use the $z$ component of the translation to initialize each laser scanner's vertical offset, $h_{i}$ [see (9)], and the yaw component of the rotation to initialize each laser scanner's $\theta_{o i}$ [see (8)].

We then formulate the following constrained minimization problem to enforce (8) and (9):

$$
\begin{aligned}
& \min \sum_{i, j, k}\left[\alpha_{i}\left(\rho_{i j k}+\rho_{o i}\right){ }^{C} \overline{\mathbf{n}}_{j}^{T} L_{i}^{C} \mathbf{C}^{L_{i}} \overline{\mathbf{p}}_{i j k}+{ }^{C} \overline{\mathbf{n}}_{j}^{T}{ }^{C} \mathbf{t}_{L_{i}}-d_{j}\right]^{2} \\
& \text { s. t. }{ }_{L_{i}}^{C} \mathbf{C}={ }_{L}^{C} \mathbf{C} \mathbf{C}_{z}\left(\theta_{o i}\right), \quad{ }_{L}^{C} \mathbf{C}^{T}\left({ }^{C} \mathbf{t}_{L_{i}}-{ }^{C} \mathbf{t}_{L}\right)=\left[\begin{array}{lll}
0 & 0 & h_{i}
\end{array}\right]^{T}
\end{aligned}
$$

where the optimization variables are $\alpha_{i}, \rho_{o i}, \theta_{o i}, h_{i}, i=2, \ldots, 64, \alpha_{1}, \rho_{o 1},{ }^{C} \mathbf{t}_{L}$, and ${ }_{L}^{C} \mathbf{C}$. ${ }^{4}$ Note that the constraints in (22) should be taken into account using the method of Lagrange multipliers. For the implementation details of the Levenberg-Marquardt algorithm we refer the interested reader to [8].

\subsection{Observability Conditions}

In this section, we examine the conditions under which the unknown lidar-Ladybug transformation and the intrinsic parameters of the lidar are observable, and thus can be estimated using the algorithms in Sections 2.1 to 2.4.

\subsubsection{Observation of One Plane}

Suppose we are provided with lidar measurements that lie only on one plane whose normal vector is denoted as ${ }^{C} \overline{\mathbf{n}}_{1}$. In this case, it is easy to show that the measurement constraint in (6) does not change if ${ }_{L}^{C} \mathbf{C}$ is perturbed by a rotation around ${ }^{C} \overline{\mathbf{n}}_{1}$, represented by the rotation matrix $\mathbf{C}^{\prime}$ :

$$
{ }^{C} \overline{\mathbf{n}}_{1}^{T} \mathbf{C}_{L_{i}}^{\prime C} \mathbf{C}^{L_{i}} \mathbf{p}_{i 1 k}+{ }^{C} \overline{\mathbf{n}}_{1}^{T C} \mathbf{t}_{L_{i}}-d_{1}=0 \Longrightarrow{ }^{C} \overline{\mathbf{n}}_{1 L_{i}}^{T C} \mathbf{C}^{L_{i}} \mathbf{p}_{i 1 k}+{ }^{C} \overline{\mathbf{n}}_{1}^{T C} \mathbf{t}_{L_{i}}-d_{1}=0
$$

\footnotetext{
${ }^{4}$ In general, the optimization should be performed over $\phi_{i}$ as well. However, in our experiments, we observed that the provided value of $\phi_{i}$ by the manufacturer is sufficiently accurate, and thus excluded it from the calibration.
} 
The second equation is obtained from the first, since ${ }^{C} \overline{\mathbf{n}}_{1}$ is an eigenvector of $\mathbf{C}^{\prime}$, thus ${ }^{C} \overline{\mathbf{n}}_{1}^{T} \mathbf{C}^{\prime}={ }^{C} \overline{\mathbf{n}}_{1}^{T}$. Therefore, when observing only one plane, any rotation around the plane's normal vector is unobservable. Similarly, if we perturb ${ }^{C} \mathbf{t}_{L_{i}}$ by a translation parallel to the plane, represented by $\mathbf{t}^{\prime}$, the measurement constraint does not change:

$$
{ }^{C} \overline{\mathbf{n}}_{1 L_{i}}^{T C} \mathbf{C}^{L_{i}} \mathbf{p}_{i 1 k}+{ }^{C} \overline{\mathbf{n}}_{1}^{T}\left({ }^{C} \mathbf{t}_{L_{i}}+\mathbf{t}^{\prime}\right)-d_{1}=0 \Longrightarrow{ }^{C} \overline{\mathbf{n}}_{1 L_{i}}^{T C} \mathbf{C}^{L_{i}} \mathbf{p}_{i 1 k}+{ }^{C} \overline{\mathbf{n}}_{1}^{T C} \mathbf{t}_{L_{i}}-d_{1}=0 .
$$

This relationship holds since ${ }^{C} \overline{\mathbf{n}}_{1}^{T} \mathbf{t}^{\prime}=0$. Therefore, when observing only one plane, any translation parallel to the plane's normal is unobservable.

\subsubsection{Observation of Two Planes}

Consider now that we are provided with measurements from two planes, described by ${ }^{C} \overline{\mathbf{n}}_{1}, d_{1},{ }^{C} \overline{\mathbf{n}}_{2}, d_{2}$. If we perturb the laser scanner's relative translation with $\mathbf{t}^{\prime \prime} \propto{ }^{C} \overline{\mathbf{n}}_{1} \times{ }^{C} \overline{\mathbf{n}}_{2}$ [see (24)], none of the measurement constraints will change, since ${ }^{C} \overline{\mathbf{n}}_{1}^{T} \mathbf{t}^{\prime \prime}={ }^{C} \overline{\mathbf{n}}_{2}^{T} \mathbf{t}^{\prime \prime}=0$. Therefore, we conclude that the relative translation cannot be determined if only two planes are observed.

\subsubsection{Observation of Three Planes}

In this section, we prove that when three planes with linearly independent normal vectors are observed, we can determine all the unknowns. For this purpose, we first determine the relative orientation ${ }_{L_{i}}^{C} \mathbf{C}$ and the offset $\rho_{o i}$ and then find the scale $\alpha_{i}$ and relative translation ${ }^{C} \mathbf{t}_{L_{i}}$. Let us assume that the $i$-th laser scanner has measured four points on each plane, denoted as $\left(\rho_{i j k},{ }^{L_{i}} \overline{\mathbf{p}}_{i j k}\right), j=1,2,3, k=1, \ldots, 4$. Each of these points provides one constraint of the form (7). We first eliminate the unknown relative translation and scale, by subtracting the constraints for point $k=1$ from $k=2$, point $k=2$ from $k=3$, and point $k=3$ from $k=4$, and obtain:

$$
\begin{aligned}
{ }^{C} \overline{\mathbf{n}}_{j L_{i}}^{T C} \mathbf{C}\left(\mathbf{u}_{j 12}^{i}+\rho_{o i} \mathbf{v}_{j 12}^{i}\right) & =0 \\
{ }^{C} \overline{\mathbf{n}}_{j L_{i}}^{T} \mathbf{C}\left(\mathbf{u}_{j 23}^{i}+\rho_{o i} \mathbf{v}_{j 23}^{i}\right) & =0 \\
{ }^{C} \overline{\mathbf{n}}_{j L_{i}}^{T} \mathbf{C}\left(\mathbf{u}_{j 34}^{i}+\rho_{o i} \mathbf{v}_{j 34}^{i}\right) & =0
\end{aligned}
$$

where $\mathbf{u}_{j k l}^{i} \triangleq \rho_{i j k}{ }^{L_{i}} \overline{\mathbf{p}}_{i j k}-\rho_{i j l}{ }^{L_{i}} \overline{\mathbf{p}}_{i j l}, \mathbf{v}_{j k l}^{i} \triangleq{ }_{i} \overline{\mathbf{p}}_{i j k}-{ }^{L_{i}} \overline{\mathbf{p}}_{i j l}$, and $j=1,2,3$. Note that ${ }^{L_{i}} \overline{\mathbf{p}}_{i j k}$ and ${ }^{L_{i}} \overline{\mathbf{p}}_{i j l}$ lie on the intersection of the unit sphere and the cone specified by the beams of the $i$-th laser scanner. Since the intersection of a co-centric unit sphere and a cone is always a circle, we conclude that all $\mathbf{v}_{j k l}^{i}$ for a given $i$ belong to a plane and have only two degrees of freedom. Thus, we can write $\mathbf{v}_{j 34}^{i}$ as a linear combination of $\mathbf{v}_{j 12}^{i}$ and $\mathbf{v}_{j 23}^{i}$, i.e.,

$$
\mathbf{v}_{j 34}^{i}=a \mathbf{v}_{j 12}^{i}+b \mathbf{v}_{j 23}^{i}
$$


for some known scalars $a$ and $b$. Substituting this relationship in (27), and using (25)-(26) to eliminate the terms containing $\rho_{o i}$ yields:

$$
{ }^{C} \overline{\mathbf{n}}_{j L_{i}}^{T C} \mathbf{C}\left(\mathbf{u}_{j 34}^{i}-a \mathbf{u}_{j 12}^{i}-b \mathbf{u}_{j 23}^{i}\right)=0
$$

for $j=1,2,3$. The only unknown in this equation is the relative orientation ${ }_{L_{i}}^{C} \mathbf{C}$ of the $i$-th laser scanner. These equations are identical to those for orientation estimation using line-to-plane correspondences, which is known to have at most eight solutions that can be analytically computed when ${ }^{C} \overline{\mathbf{n}}_{j}, j=1,2,3$, are linearly independent [4]. Once ${ }_{L_{i}}^{C} \mathbf{C}$ is known, we can use any of (25)-(27) to compute the offset $\rho_{o i}$. Finally, the scale and the relative translation can be obtained from (21).

\section{Experiments}

In order to validate the proposed calibration method, we conducted a series of experiments. Specifically, we rigidly connected a Velodyne 3D lidar and a Ladybug2 spherical vision system, and recorded measurements of a 36 " $\times 40$ " calibration plane with 16 fiducial markers at 40 different configurations. By processing the Ladybug's images using a PnP algorithm followed by a least-square refinement [9], we computed the normal vector and the distance of the calibration plane at each configuration. We then identified the approximate location of the calibration plane in the lidar scans based on a coarse prior estimate for the relative rotation of the Velodyne and the Ladybug. Within these approximate locations, we detected the lidar data points reflected from the calibration plane, based on their depth discontinuity.

Once the Velodyne's measurements for each configuration of the calibration plane were available, we used the methods described in Sections 2.1-2.4 to accurately estimate the lidar's intrinsic parameters and the lidar-camera transformation. Note, however, that in order to increase the robustness of our algorithm to outliers, we did not directly use the raw laser points measured by the lidar. Instead, for each laser scanner, we fit small line segments to the intersection of the laser scanner's beam and the calibration plane, and used the endpoints of these line segments as the lidar's measurements. ${ }^{5}$

We compare the accuracy of the estimated intrinsic lidar parameters with those provided by the manufacturer. For this purpose, we transform the raw lidar measurements to the lidar's Euclidean frame [see (2)] using both the estimated and the manufacturer-provided intrinsic parameters. We then fit planes to the lidar points belonging to the calibration planes and use the residual fitting error to evaluate the accuracy of the estimated intrinsic parameters. In Fig. 3, the histogram of these residual errors for the manufacturer-provided and the estimated intrinsic parameters are shown, clearly demonstrating the superior accuracy of our method.

\footnotetext{
${ }^{5}$ Note that in general the intersection of the cone induced by the laser scanner's beam with a plane results in a conic section, and not a straight line. However, in practical situation this conic section can be approximated with a sequence of straight line segments.
} 


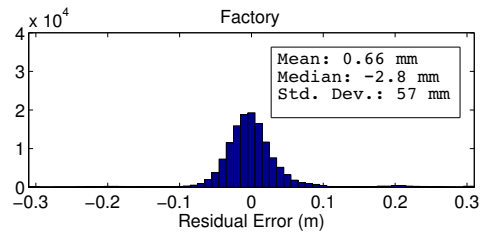

(a)

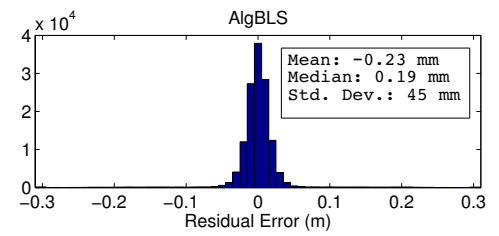

(b)

Fig. 3 Histograms of the residual error for the lidar points belonging to 40 planar surfaces using the intrinsic lidar parameters from: (a) the manufacturer; (b) the proposed method.

To further evaluate the performance of our calibration algorithm, we created photorealistic reconstructions of several indoor and outdoor scenes from the University of Minnesota campus (see Fig. 4). For each scene, the raw measurements of the lidar are first transformed to Euclidean coordinates using the estimated intrinsic parameters of the lidar and then they are expressed in the Ladybug's frame of reference. In the next step, the lidar points are overlaid on the spherical image provided by the camera to associate them with an image pixel. Note, however, that after this step many of the image pixels will not be associated with any lidar points due to the low resolution of the lidar scans compared to the Ladybug's images. We assigned such "orphan" pixels a 3D point obtained through linear interpolation of the surrounding lidar points. The final result is a set of image pixels with 3D coordinates (i.e., 3D pixels). Finally, we converted the 3D pixels to 3D surfaces using Delaunay triangulation [2]. In Fig. 4, a selection of the reconstructed surfaces are shown for indoor and outdoor scenes. Note that white gaps in the reconstructed surfaces result from missing lidar measurements due to occlusion or specular reflection of the laser beams from glass and shiny surfaces.

\section{Conclusions and Future Work}

In this paper, we presented a novel method for intrinsic calibration of a Velodyne 3D lidar and extrinsic calibration with respect to a camera. Specifically, we developed an analytical method for computing a precise initial estimate for both the lidar's intrinsic parameters and the lidar-camera transformation. Subsequently, we used these estimates to initialize an iterative nonlinear least-squares refinement of all the calibration parameters. Additionally, we presented an observability analysis to determine the minimal conditions under which it is possible to estimate the calibration parameters. Experimental results from both indoor and outdoor scenes are used to demonstrate the achieved accuracy of the calibration process by photorealistic reconstruction of the observed areas. Optimally combining multiple images and lidar scans over consecutive time steps for mapping large areas while at the same 
time increasing the 3D points' resolution and revealing occluded areas, is part of our ongoing research work.

\section{References}

1. Auzinger, W., Stetter, H.J.: An elimination algorithm for the computation of all zeros of a system of multivariate polynomial equations. In: Proc. Int. Conf. on Numer. Math., pp. 1130. Singapore (1988)

2. de Berg, M., Cheong, O., van Kreveld, M., Overmars, M.: Computational Geometry: Algorithms and Applications. Springer-Verlag (2008)

3. Byröd, M., Josephson, K., Åström, K.: A column-pivoting based strategy for monomial ordering in numerical Gröbner basis calculations. In: Proc. Euro. Conf. Comput. Vision, pp. 130-143. Marseille, France (2008)

4. Chen, H.H.: Pose determination from line-to-plane correspondences: existence condition and closed-form solutions. IEEE Trans. Pattern Anal. Mach. Intell. 13(6), 530-541 (1991)

5. Cox, D., Little, J., O'Shea, D.: Using Algebraic Geometry. Springer (2004)

6. Naroditsky, O., Patterson IV, A., Daniilidis, K.: Automatic alignment of a camera with a line scan lidar system. In: Proc. IEEE Int. Conf. Robot. Autom. Shanghai, China (2011)

7. Pandey, G., McBride, J., Savarese, S., Eustice, R.: Extrinsic calibration of a 3d laser scanner and an omnidirectional camera. In: Proc. IFAC Symp. on Intell. Auton. Veh. Lecce, Italy (2010)

8. Press, W.H., Teukolsky, S.A., Vetterling, W.T., Flannery, B.P.: Numerical Recipes in C. Cambridge University Press, Cambridge (1992)

9. Quan, L., Lan, Z.D.: Linear n-point camera pose determination. IEEE Trans. Pattern Anal. Mach. Intell. 21, 774-780 (1999)

10. Reid, G., Zhi, L.: Solving polynomial systems via symbolic-numeric reduction to geometric involutive form. J. Symb. Comput. 44(3), 280 - 291 (2009)

11. Scaramuzza, D., Harati, A., Siegwart, R.: Extrinsic self calibration of a camera and a 3d laser range finder from natural scenes. In: Proc. IEEE/RSJ Int. Conf. Intell. Robots Syst., pp. 4164 -4169. San Diego, USA (2007)

12. Shuster, M.D.: A survey of attitude representations. J. Astronaut. Sci. 41(4), 439-517 (1993)

13. Stamos, I., Liu, L., Chen, C., Wolberg, G., Yu, G., Zokai, S.: Integrating automated range registration with multiview geometry for the photorealistic modeling of large-scale scenes. Int. J. Comput. Vision 78(2), 237-260 (2008)

14. Trawny, N., Roumeliotis, S.I.: On the global optimum of planar, range-based robot-to-robot relative pose estimation. In: Proc. IEEE Int. Conf. Robot. Autom. Anchorage, AK (2010)

15. Trawny, N., Zhou, X.S., Roumeliotis, S.I.: 3D relative pose estimation from six distances. In: Proc. Robot. Sci. Syst. Seattle, WA (2009)

16. Unnikrishnan, R., Hebert, M.: Fast extrinsic calibration of a laser rangefinder to a camera. Tech. rep., Carnegie Mellon University, Robotics Institute (2005)

17. Zhang, Q., Pless, R.: Extrinsic calibration of a camera and laser range finder (improves camera calibration). In: Proc. IEEE/RSJ Int. Conf. Intell. Robots Syst., pp. 2301-2306. Sendai, Japan (2004) 


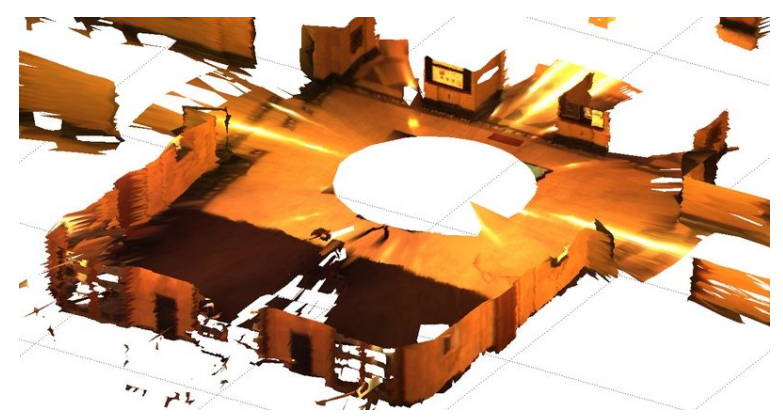

(a)

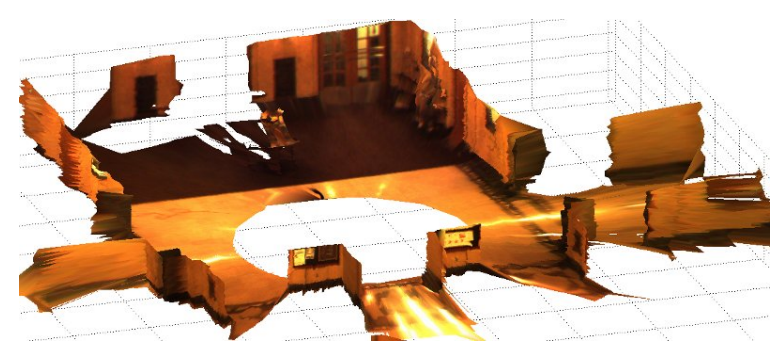

(b)

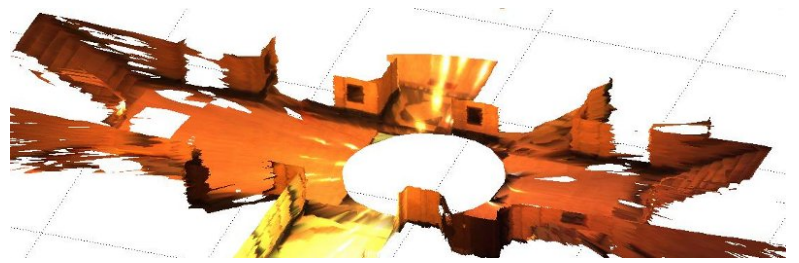

(c)

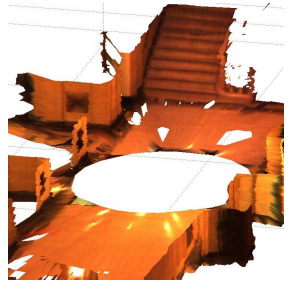

(d)

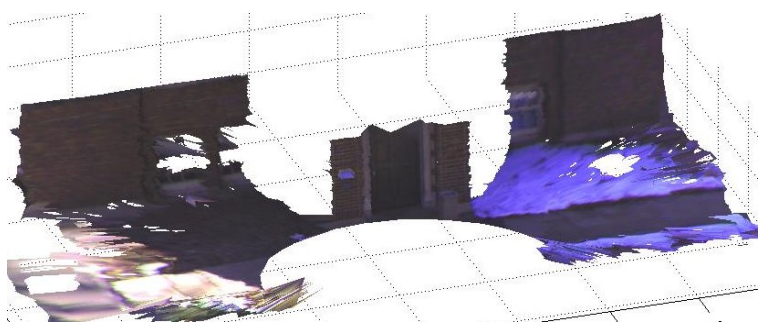

(e)

Fig. 4 A selection of the results from photorealistic reconstruction of indoor and outdoor scenes (best viewed in color). The white gaps are the regions where at least one of the sensors did not return meaningful measurements (e.g., due to occlusion, specular reflections, or limited resolution and field of view). Note that the depth of the scene can be inferred from the dotted grids. $(a, b)$ : Center of a building with several corridors, viewed from different directions; (c,d): A indoor scene containing two stair cases, viewed from two different directions; (e) An outdoor scene with snow on the ground. 